\title{
Comparison of Abdominal and Lumbar Muscles Electromyography Activity During Two Types of Stabilization Exercises
}

\author{
Farahnaz Emami ${ }^{1}$; Soraya Pirouzi ${ }^{1,{ }^{*}}$; Shohreh Taghizadeh ${ }^{1}$ \\ ${ }^{1}$ Department of Physiotherapy, Center for Human Sciences Research, Faculty of Rehabilitation Sciences, Shiraz University of Medical Sciences, Shiraz, IR Iran \\ ${ }^{*}$ Corresponding author: Soraya Pirouzi, Department of Physiotherapy, Center for Human Sciences Research, Faculty of Rehabilitation Sciences, Shiraz University of Medical Sciences, \\ Shiraz, IR Iran. E-mail: piroozis@sums.ac.ir
}

Received: September 8, 2013; Accepted: December 4, 2013

\begin{abstract}
Background: Stabilization exercises lead to improve muscle performance and can be used to prevent and treat of low back pain. Objectives: Aim of the present study is to evaluate and compare the level of abdominal and lumbar muscles activity during two types of stabilization exercises.

Patients and Methods: Thirty healthy females aged between 20-30 years old participated in this quasi-experimental study. Muscle activity was recorded from Transversus Abdominus (TrA), Internal Oblique(IO) and multifidus on both sides with electromyography (EMG) device during the quadruped and dead bug exercises. EMG data were normalized to the percentage of maximum voluntary isometric contraction and paired t-test was used for statistical analysis.

Results: The mean muscle activity in the right arm reach of quadruped and dead bug exercises showed that right IO and TrA muscles produced greater activity during quadruped exercise $(\mathrm{P}=0.015, \mathrm{P}=0.001$, respectively). The comparison between the mean muscles activity in the left leg reach of two exercises showed that right and left TrA muscles produced greater activity during left leg reach in quadruped exercise $(\mathrm{P}=0.005, \mathrm{P}=0.046$, respectively). Also, the comparison between the mean muscle activity in simultaneous movements of left leg and right arm reach in the 2 exercises showed that right TrA muscle was activated at a higher level compared to the other muscles in the quadruped exercise $(\mathrm{P}=0.002)$.

Conclusions: The results showed quadruped exercise can provide stability, coordination, and smoothness of the movements in healthy subjects and all considered muscles participated in this exercise.
\end{abstract}

Keywords: Electromyography; Stabilization Exercises; Muscle, Skeletal

\section{Background}

Stability and mobility are two important elements in the vertebral column. Joint stability is an effective adaptation of the joints to each specific load demand. During different conditions' gravity, muscles and ligamentous forces can produce joint compression which results in spinal stability [1-6] Complex loading patterns act on the passive structures of osteo-ligamentous spine and, if unprotected, the lumbar spine is vulnerable to being impaired [7]. The other main factor which challenges the stability is movement [1-4]. Spinal stability is required for appropriate movements and unstable lumbar spine could contribute to tissue strain injury and result in chronic back pain [7].

LBP (low back pain) is a common problem in today's industrial societies and a major cause of disability and sickness absence $[8,9]$. In general population, the prevalence of chronic LBP has been reported as $10-30 \%$ In addition, LBP is more common in females compared to males [8,10-12]. Several studies have confirmed that function and co-operation of the stabilizing muscles of the lumbar spine are often impaired in the patients with LBP [13-16]. Therefore, muscles play an important role in the etiology, presenta- tion, and treatment of low back disorders. Besides, stabilizing exercises decrease trunk muscles impairments and restore the ability of these muscles to provide lumbar joint protection $[13,16]$.

Up to now, a large number of studies have been conducted on the role of the muscles in stabilizing the vertebral column [17-19]. For example, the activities of the rectus abdominis, transverse abdominis (TrA) and internal oblique (IO) and external oblique muscles were investigated during abdominal hallowing in four starting positions. The results suggested that all the four positions could facilitate $\mathrm{Tr}$, IO and rectus abdominis activities, while the external oblique muscles had minimal activity [20]. Also, McGill and Karpowicz noticed that abdominal bracing enhanced the activation of obliques muscles [21]. Another study showed an increased activity of both local and global muscles during stabilization exercises on unstable surfaces [22]. Furthermore, Pinto et al. noticed that TrA and IO muscles' thickness changed during lower limb tasks [23]. Also, some studies showed that trunk muscles activity increased during abdominal bracing and Swiss ball exercise performed in the pool $[24,25]$.

Copyright (C) 2015, Zahedan University of Medical Sciences. This is an open-access article distributed under the terms of the Creative Commons Attribution-NonCommercial 4.0 International License (http://creativecommons.org/licenses/by-nc/4.0/) which permits copy and redistribute the material just in noncommercial usages, provided the original work is properly cited. 


\section{Objectives}

Previous studies demonstrated that trunk muscles could be activated during different activities. Therefore, the present study aims to compare the level of contraction of the abdominal and lumbar muscles in order to clarify the role of trunk core muscles' activity during two types of stabilization exercises. The effect of the upper and lower extremities movements on the trunk muscles' activity is evaluated, as well. Clinical purpose of the present study is improving core muscles activity and prevention of low back injuries in future.

\section{Patients and Methods}

This quasi-experimental study was carried out in the research center of Shiraz Rehabilitation Department, Shiraz University of Medical Sciences, Shiraz, Iran. The study was conducted on 30 healthy right handed females aging between 20 and 30 years old who had no known neuromuscular, orthopedic, or cardiovascular conditions. Considering $\mathrm{d}=4, \mathrm{SD}=11, \alpha=0.05$ and $\beta=0.2$, the sample size was calculated from the (1- $\rho) \mathrm{N}$ and simple sampling was used. After that, 24 subjects were selected for this study. In order to increase the accuracy of the results, 30 subjects were recruited into the study. All the subjects signed written informed consents for taking part in the study and had no previous experience of stabilization exercises. The subjects with any past recurrent LBP, BMI (body mass index) above 27, current neurologic deficits, and pain or disability of the upper or lower limbs as well as the left handed ones were excluded from the study.

\subsection{Instrument}

The study data were collected using Mega win (Mega electronics) LTD (made in Finland; v. 2.5 a 16). EMG signals were recorded using 6 pairs of self-adhesive disposable disc surface electrodes (Medico-lead lok) with an electrical contact of $1 \mathrm{~cm}^{2}$ and a centre-to-centre distance of $2 \mathrm{~cm}$. The electrodes were placed bilaterally over the muscle bellies of TrA as in the studies conducted by Vezina and HubleyKozey [26], multifidus as in the studies performed by Stevens et al. and IO as in Stevens' study [5]. In addition, the skin was prepared and the skin resistance was decreased to lower than 5 Kilo Ohms. Prior to processing the raw EMG data, a customized quality control program in conjunction with visual inspection was used on all the channels in order to detect and eliminate the possible contamination of the EMG signal by heartbeat and other artifacts. The EMG data were amplified and fully rectified by band pass filter at $5-500 \mathrm{~Hz}$ and then sampled at $1000 \mathrm{~Hz}$. Then, the data were recorded onto a hard disk and transferred to floppy disks for offline processing.

The electrode sites were validated using manual muscle testing and doing MVC to isolate each muscle activity and decrease cross talk [26]. For minimizing the noise, each channel had an isolated ground electrode and for preventing the artifact, the electrodes were well taped. To en- sure a stable temperature and impedance, no recording was made within 10 minutes of electrode placement. The subjects were asked to relax completely in the supine position and the noise of the channels was less than $5 \mathrm{~K} \Omega$.

\subsection{Data Collection}

Each subject performed three different trials in order to determine the mean of the maximal effort of the target muscles. To evaluate the maximal voluntary contraction (MVC) for TrA, all the subjects were in crook lying position with flexed knees, flat feet, and hips flexed to $70^{\circ} \mathrm{C}$ measured with a goniometer. Then, they were instructed to hollow in and bring their umbilicus up toward the spine and maintain this position for 5 seconds. The exercise performance was closely monitored to ensure that the subjects were not tilting the pelvis backward or inhaling and elevating the rib cage to make the abdomen look flat. The subjects performed 3 successive trials of each exercise with a short rest of approximately $1 \mathrm{~min}$ between each trial to prevent fatigue [26]. In order to measure the MVC of the IO muscles in the sitting position, the hips and the chest were fixed by two straps and the subjects were asked to produce maximal rotation without flexion toward right and left sides and then 3 trials of this exercise were performed. A pause of $1 \mathrm{~min}$ was allowed between the trials [17].

For determining the MVC of the multifidus muscles in prone position, the lower extremities and the chest were fixed with two straps. The subjects performed maximal trunk extension against resistance for 3 times. A 1 min pause was also allowed between the trials [26].

After measurement of MVCs, all the subjects performed two types of stabilization exercises; i.e. quadruped and dead bug exercises. To ensure that the subjects performed the exercises in the right way, they were familiarized with the exercises in two sessions. The quadruped exercise was performed in quadruped position with movements of the extremities being executed in a random sequence. At the beginning of each exercise, a neutral spine position was demonstrated by the examiner and the subjects were encouraged to hold this position during the whole course of the exercise. The neutral position was set about halfway between the full extension and the flat position of the spine. In the right arm reach stage, the subjects contracted the TrA muscles, maintained this contraction, and lifted the right upper extremity to a horizontal level for 5 seconds and then 3 trials were performed. A 15 seconds pause was also allowed between the trials (Figure 1). In the next stage, the subjects contracted the TrA muscles and extended the left lower extremity to a horizontal level. During the static phase, the leg was held in the extended position for 5 seconds (Figure 2).

Afterwards, left leg extension was coupled with simultaneous rising of the right arm to the horizontal level (Birddog) and during the static phase, this position was held for 5 seconds and registration was done (Figure 3). For dead bug exercise, the subjects were in supine position with left knee flexed in $70^{\circ} \mathrm{C}$ and right lower extremity completely 
extended. In the right arm reach stage, the subjects contracted the TrA muscles, maintained this contraction, and lifted the right upper extremity over the head level for 5 seconds and then 3 trials were performed. A 15 seconds pause was also allowed between the trials (Figure 4).

In the next stage, the subjects contracted the TrA muscles and flexed the left lower extremity to 90 - 90 position. During the static phase, the leg was held in the flexed position for 5 seconds (Figure 5). In the final stage, left leg flexion was coupled with simultaneous depressing of the right arm and during the static phase, this position was held for 5 seconds and registration was done (Figure 6).

In this study, band pass filter of $10-1000 \mathrm{~Hz}$ and manual muscle testing were used to confirm the sites of the electrodes and eliminate the cross talk resulting from the other muscles. Since analyzing the data obtained from both sites was difficult and the subjects were right handed, the right hand and the left leg were selected for motion analysis.

For EMG amplitude analysis, artifact-free, raw EMG sections were used. The recorded data were fully rectified and smooth. For each of the muscles, the root mean square amplitudes were calculated for the normalization trials by a computer algorithm which determined 500 consecutive samples ( 0.5 second) of raw EMG within 5 seconds. The mean MVC was used to provide a basis for EMG signal amplitude normalization and the mean normalized EMG values were calculated. During the test trials, a computer program separated the 2 phases based on an event marker. Also, Root Mean Square (RMS) was separately calculated for static and dynamic phases.

All the statistical analyses were performed using the SPSS 16 and $\alpha=0.05$ was considered as the significance level. Paired t-test was used for comparing the three stages of quadruped and dead bug exercises. Moreover, the reliability and validity of the data were confirmed through a pilot study. This pilot study aimed to determine the repeatability of the data and revealed ICCs of $0.66-0.87$ indicating the high reliability of the data.

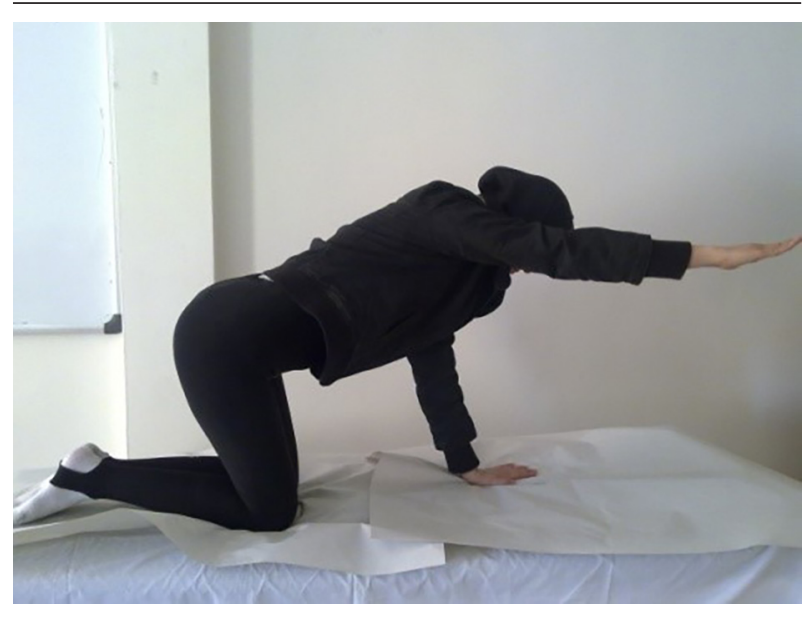

Figure 1. Right Arm Reach Position

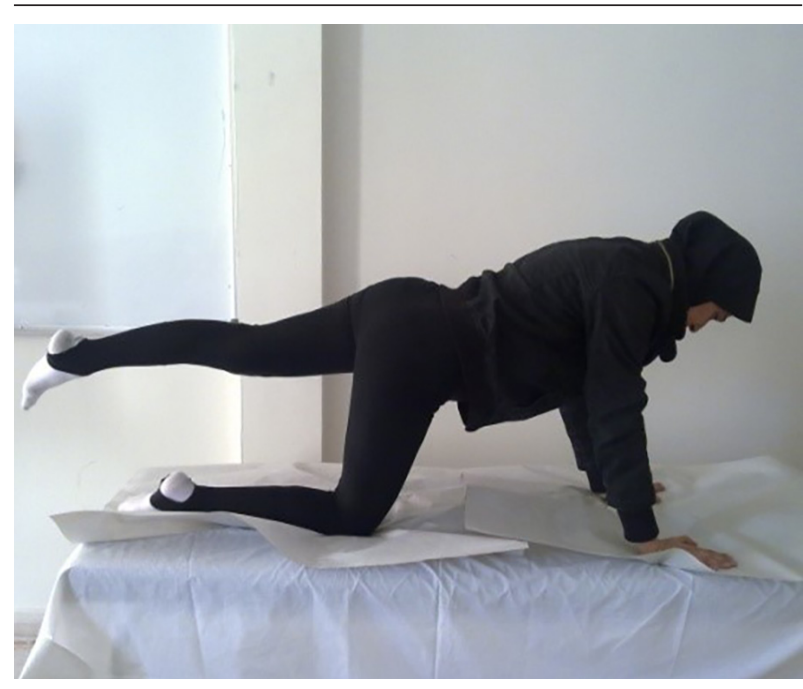

Figure 2. Left Leg Reach Position

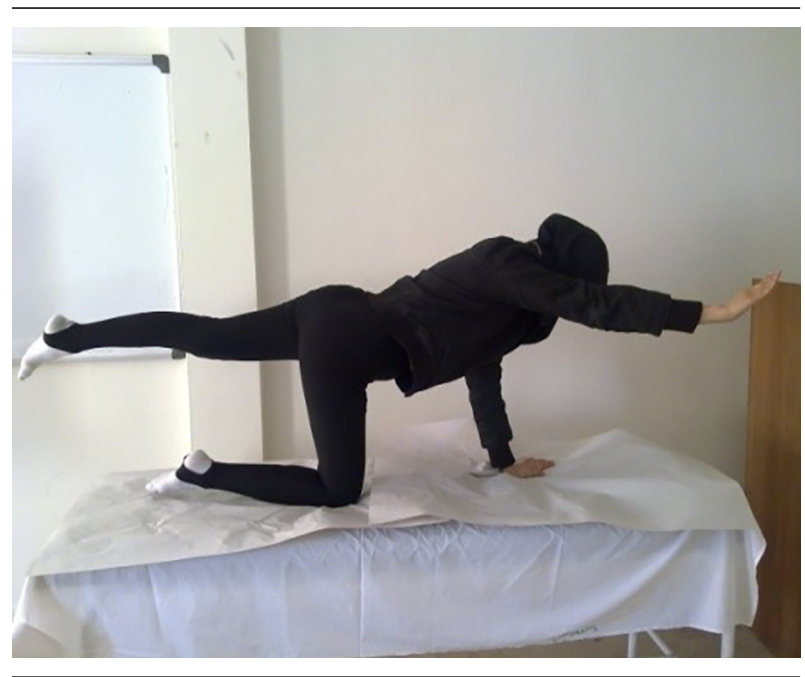

Figure 3. Birddog Position

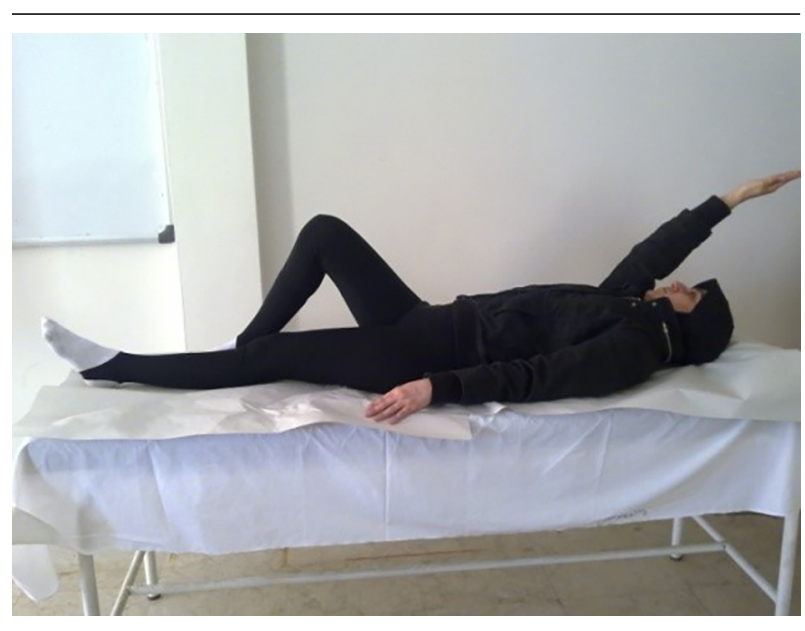

Figure 4. Right Arm Reach Position 


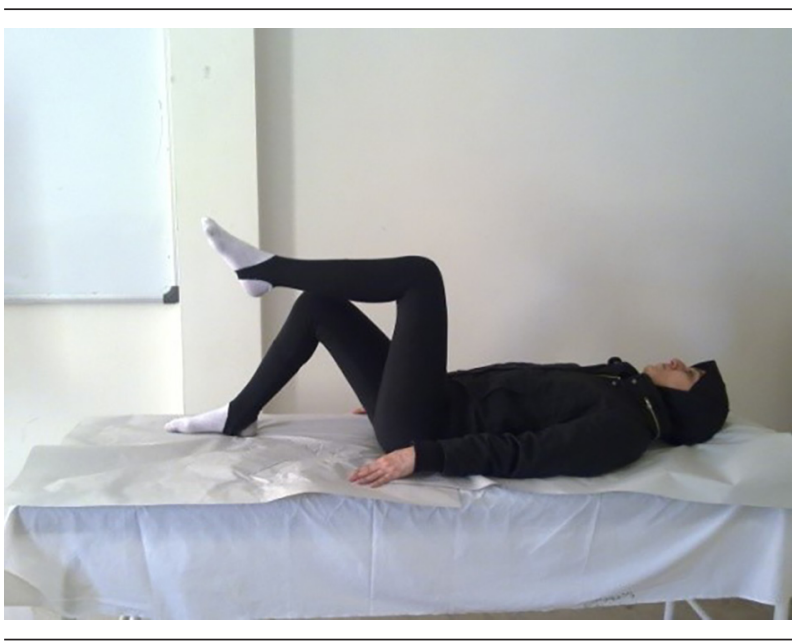

Figure 5. Left Leg Reach Position

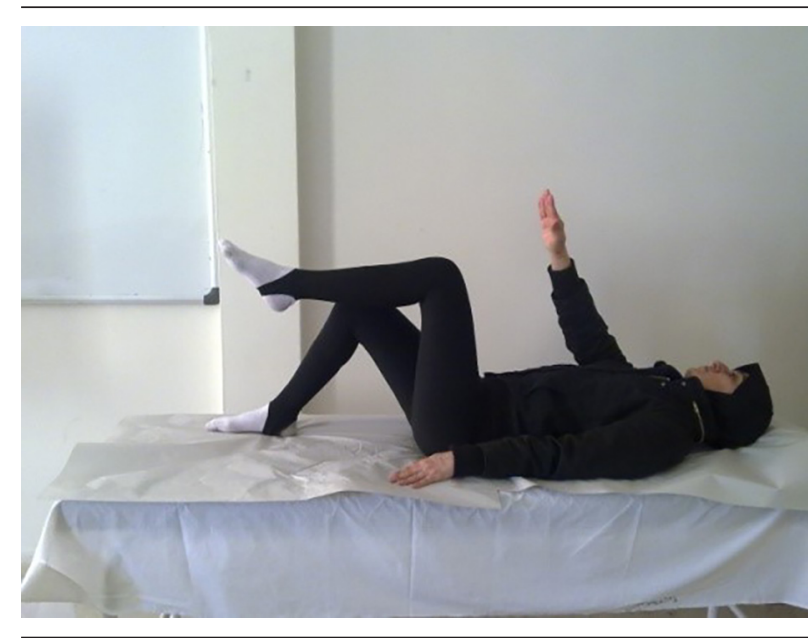

Figure 6. Simultaneous Movement of Left Leg Reach and Right Arm Reach

Table 1. Comparison of Mean Muscles Activation While Right Arm Reach in Quadruped and Dead Bug Exercises a,b

\begin{tabular}{|c|c|c|c|}
\hline Variables & Quadruped, mv & Dead Bug, mv & P-Value \\
\hline \multicolumn{4}{|l|}{ Rt IO } \\
\hline Right arm reach & $0.62 \pm 1.23$ & $0.59 \pm 92.0$ & $0.015^{c}$ \\
\hline Left leg reach & $0.69 \pm 1.27$ & $0.76 \pm 1.11$ & 0.235 \\
\hline \multicolumn{4}{|l|}{ Lt IO } \\
\hline Right arm reach & $1.51 \pm 1.29$ & $0.72 \pm 1.35$ & 0.844 \\
\hline Left leg reach & $1.36 \pm 1.76$ & $0.9 \pm 1.52$ & 0.275 \\
\hline \multicolumn{4}{|l|}{ Rt TrA } \\
\hline Right arm reach & $2.14 \pm 2.57$ & $0.56 \pm 1.13$ & $0.001^{\mathrm{c}}$ \\
\hline Left leg reach & $1.75 \pm 2.58$ & $1.26 \pm 1.45$ & $0.005^{c}$ \\
\hline \multicolumn{4}{|l|}{ Lt TrA } \\
\hline Right arm reach & $1.38 \pm 1.60$ & $0.55 \pm 1.20$ & 0.08 \\
\hline Left leg reach & $1.69 \pm 2.08$ & $0.87 \pm 1.44$ & $0.046^{c}$ \\
\hline \multicolumn{4}{|l|}{ RT MF } \\
\hline Right arm reach & $0.55 \pm 0.31$ & $4.36 \pm 1.31$ & 0.173 \\
\hline Left leg reach & $0.51 \pm 0.55$ & $0.95 \pm 0.42$ & 0.475 \\
\hline \multicolumn{4}{|l|}{ Lt MF } \\
\hline Right arm reach & $0.85 \pm 0.48$ & $4.29 \pm 1.26$ & 0.245 \\
\hline Left leg reach & $1.38 \pm 0.64$ & $1.38 \pm 0.54$ & 0.655 \\
\hline
\end{tabular}

a Abbreviations: IO: Internal Oblique, Lt: Left, MF: Multifidus, Rt: Right,. TrA: Transeverse Abdominus.

$\mathrm{b}=30$.

c Statistically significant.

\section{Results}

In this study, the participants' mean age, mean height, and mean weight was $23.1 \pm 2.41$ years, $163.80 \pm 5.42 \mathrm{~cm}$ and $54.3 \pm 8.38 \mathrm{~kg}$, respectively. According to the results of paired t-test, a statistically significant difference was observed between the right arm reach of quadruped and dead bug exercises ( $\mathrm{P}=0.015, \mathrm{P}=0.01$, respectively). In other words, right IO and TrA muscles had higher activities in comparison to the other muscles in quadruped exercise (Table 1). According to the results of the paired t-test, activity of TrA muscles in left leg reach during the quadruped exercise was higher than the same position in the dead bug exercise (in right $\operatorname{Tr} \mathrm{A}, \mathrm{P}=0.005$, in left side, $\mathrm{P}$ $=0.046)$ (Table 1 ). Also, the activity of the right TrA muscle was higher when simultaneous movements of the left leg and right arm were performed in the quadruped exercise compared to the dead bug exercise $(\mathrm{P}=0.002)$ (Table 2$)$. 
Table 2. Comparison of Mean Muscles Activation During the Simultaneous Movement of Extremities in Quadruped and Dead Bug Exercises a,b

\begin{tabular}{lccc}
\hline Variables & Quadruped, mv & Dead Bug, mv & P-Value \\
\hline Rt IO & $0.8 \pm 1.35$ & $0.75 \pm 1.13$ & 0.147 \\
Lt IO & $1.63 \pm 1.90$ & $0.67 \pm 1.48$ & 0.173 \\
Rt TrA & $2.63 \pm 3.11$ & $0.93 \pm 1.45$ & $0.002^{\mathrm{C}}$ \\
Lt TrA & $1.55 \pm 1.95$ & $1.76 \pm 1.62$ & 0.425 \\
\hline Rt MF & $0.52 \pm 0.76$ & $2.8 \pm 0.82$ & 0.898 \\
Lt MF & $0.86 \pm 1.01$ & $5.41 \pm 1.24$ & 0.790 \\
\hline
\end{tabular}

a Abbreviations: IO: internal oblique, Lt: Left, MF: multifidus, Rt: Right, TrA: Transeverse abdominus.

$\mathrm{b} \mathrm{n}=30$.

c Statistically significant.

\section{Discussion}

The results of the present study showed the mean activity of the abdominal and lumbar muscles were different in these exercises. Overall, in right arm reach of quadruped exercise, right TrA and IO had the highest activation compared to the other muscles.

In quadruped position, the abdominal muscles are stretched due to gravity and then, stretch reflex appears [27]. During right arm reach position of quadruped exercise, spinal segments rotate, the distance between the ribcage and pelvis increases and stability is disrupted. Consequently, the right IO contracts in order to prevent this instability. Also the contraction of IO muscle can preserve the spinal posture in this stage of Quadruped exercise [28].

In the other hand, unlike TrA, IO muscle is dependent to load direction into spinal column. Also due to upper limb movement as a predict perturbation, activity of this muscle can occurred and provide spinal column stability. During this task the central nervous system (CNS) predicts the effects of movement and plans muscles recruitment to overcome the perturbation. This prediction involves an internal system of body dynamics. Indeed CNS used feed forward mechanism in two different ways: 1non directional response. This response activated intrinsic muscles and minimized intersegmental motions. 2Directional specific response of global muscles to control spinal alignment. This mechanism leads to anticipatory muscles activity and then spinal curvature remains [4]. Ultrasonic images showed that IO and TrA thickness can increase during stabilization exercises [23, 29, 30]. Some studies showed that when subjects are asked to raise one of their upper limbs, greater challenge to core muscle control is achieved [31,32].

In the left leg reach of quadruped exercise, TrA muscles had the highest activation compared to the other muscles. In this position, the body weight is shifted on the other limbs and this condition can cause an imbalance in spinal segments. More effective activity of the deep abdominal muscles such as TrA muscle is necessary to maintain this position. TrA muscle performs like a lumbar corset and preserves spinal stability [30,33, 34]. Bilateral TrA activation is seen in anticipatory manner during lower limbs movement and act as a low back stabilizer prior to activities [35-37]. Also the stability in the spinal segments and sacroiliac joint can improve by increasing of tension in the thoracolumbar fascia and intra-abdominal pressure [38-40]. As mentioned before, several studies showed that muscle thickness can be considered as an indicator of muscle function. These studies suggested that activity of TrA muscle could be facilitated when the spinal stability is decreased. During the left leg reach these muscles recruited automatically in the subconscious level and compensate lack of stability. In subjects with chronic low back pain, reduction of deep abdominal muscles function and decrease of muscles thickness were seen [30,34]. Many researchers have shown that the quadruped exercise with single leg extension imposed minimal load on the spinal segments. Therefore, this stage could be prescribed in the rehabilitation program of $\operatorname{LBP}[20,31,41]$.

Comparison of the muscle activity while the simultaneous movement of the right arm and left leg in the two exercises showed that right TrA muscle activity increased in the quadruped exercise.

Moreover, it was demonstrated that the co-contraction of TrA and multifidus muscles provided stability in spinal segments. It seems that when a higher stability is needed, as in quadruped position where the base of support is narrower than usual, stronger contraction of TrA muscle is required in order to maintain lumbar stability.

One of the limitations of the present study was that it was conducted only on healthy female subjects. Further studies are required to be performed on LBP patients and the results be compared with healthy individuals. In quadruped position performed by healthy subjects, all the muscles responsible for core stability seemed to work in harmony. However, in different positions, as loads are applied to the spine and the bases of support are changed, the activity of muscles alters to provide stability. In quadruped exercise, gravity affects the muscles' activity and sensitivity of stretch receptors increase. Therefore, muscles strongly contract in this position.

\section{Acknowledgements}

The present article was extracted from the thesis written by Farahnaz Emami which was financially supported by Shiraz University of Medical Sciences, Shiraz, IR Iran (Grant No. 692). Hereby, the authors would like to thank Dr. Motealleh for his technical assistance and the statistical masters of Shiraz University of Medical Sciences. They are also grateful for the Research Improvement Center of Shiraz University of Medical Sciences and Ms. A. Keivanshekouh for improving the use of English in the manuscript. 


\section{Authors' Contributions}

All authors had equal role in design, work, statistical analysis and manuscript writing.

\section{Funding/Support}

The present article was extracted from the thesis written by Farahnaz Emami which was financially supported by Shiraz University of Medical Sciences, Shiraz, IR Iran.

\section{References}

1. Hohepa M, Scragg R, Schofield G, Kolt GS, Schaaf D. Social support for youth physical activity: importance of siblings, parents, friends and school support across a segmented school day. Int Behav Nutr Phys Act. 2007;4:54.

2. Hodges PW, Richardson CA. Contraction of the abdominal muscles associated with movement of the lower limb. Phys Ther 1997;77(2):132-42.

3. Hodges P, Cresswell A, Thorstensson A. Preparatory trunk motion accompanies rapid upper limb movement. Exp Brain Res. 1999;124(1):69-79.

4. Hodges P. Lumbopelvic stability: A functional model of the biomechanics and motor control of lumbopelvic stability. . 2 ed. Richardson C, Hodges PW, Hides J editors. Philadelphia: Churchill Livingstone; 2004.

5. Stevens VK, Vleeming A, Bouche KG, Mahieu NN, Vanderstraeten GG, Danneels LA. Electromyographic activity of trunk and hip muscles during stabilization exercises in four-point kneeling in healthy volunteers. Eur Spine J. 2007;16(5):711-8.

6. Cleland J, Schulte C, Durall C. the role of therapeutic exercise in treating instability-related lumbar spine pain: A systematic review. J Back Musculoskelet Rehabil. 2002;16(2):105-15.

7. Cholewicki J, van Dieen JH, Arsenault AB. Muscle function and dysfunction in the spine. JElectromyogr Kinesiol. 2003;13(4):303-4.

8. Horvath G, Koroknai G, Acs B, Than P, Illes T. Prevalence of low back pain and lumbar spine degenerative disorders. Questionnaire survey and clinical-radiological analysis of a representative Hungarian population. Int Orthop. 2010;34(8):1245-9.

9. Costa-Black KM, Loisel P, Anema JR, Pransky G. Back pain and work. Best Pract Res Clin Rheumatol. 2010;24(2):227-40.

10. Shiri R, Karppinen J, Leino-Arjas P, Solovieva S, Viikari-Juntura E. The association between obesity and low back pain: a meta-analysis. Am J Epidemiol. 2010;171(2):135-54.

11. Hoy D, Bain C, Williams G, March L, Brooks P, Blyth F, et al. A systematic review of the global prevalence of low back pain. Arthritis Rheum. 2012;64(6):2028-37.

12. Hoy D, Brooks P, Blyth F, Buchbinder R. The epidemiology of low back pain. Best Pract Res Clin Rheumatol. 2010;24(6):769-81.

13. Stokes IA, Gardner-Morse MG, Henry SM. Abdominal muscle activation increases lumbar spinal stability: analysis of contributions of different muscle groups. Clin Biomech (Bristol, Avon). 2011;26(8):797-803.

14. van der Hulst M, Vollenbroek-Hutten MM, Rietman JS, Hermens $\mathrm{HJ}$. Lumbar and abdominal muscle activity during walking in subjects with chronic low back pain: support of the "guarding" hypothesis? J Electromyogr Kinesiol. 2010;20(1):31-8.

15. Vasseljen O, Fladmark AM. Abdominal muscle contraction thickness and function after specific and general exercises: a randomized controlled trial in chronic low back pain patients. Man Ther 2010;15(5):482-9.

16. Freeman MD, Woodham MA, Woodham AW. The role of the lumbar multifidus in chronic low back pain: a review. $P M R J$ 2010;2(2):142-6.

17. Wagner H, Liebetrau A, Schinowski D, Wulf T, de Lussanet MH. Spinal lordosis optimizes the requirements for a stable erect posture. Theor Biol Med Model. 2012;9:13.

18. Dziuba A, Rutkowska-Kucharska A, Hadzik A, Rudzinski M, Garcarek J. Magnetic resonance imaging (MRI) for assessment of differences in geometrical parameters in muscles stabilizing vertebral column in young and older persons. Case study. Acto Bioeng Biomech. 2010;12(3):41-51.

19. Javadian Y, Behtash H, Akbari M, Taghipour-Darzi M, Zekavat H The effects of stabilizing exercises on pain and disability of patients with lumbar segmental instability. J Back Musculoskelet Rehabil. 2012;25(3):149-55.

20. Chanthapetch P, Kanlayanaphotporn R, Gaogasigam C, Chiradejnant A. Abdominal muscle activity during abdominal hollowing in four starting positions. Man Ther. 2009;14(6):642-6.

21. McGill SM, Karpowicz A. Exercises for spine stabilization: motion/motor patterns, stability progressions, and clinical technique. Arch Phys Med Rehabil. 2009;90(1):118-26.

22. Imai A, Kaneoka K, Okubo Y, Shiina I, Tatsumura M, Izumi S, et al. Trunk muscle activity during lumbar stabilization exercises on both a stable and unstable surface. J Orthop Sports Phys Ther 2010;40(6):369-75.

23. Pinto RZ, Ferreira PH, Franco MR, Ferreira MC, Ferreira ML, Teixeira-Salmela LF, et al. The effect of lumbar posture on abdomina muscle thickness during an isometric leg task in people with and without non-specific low back pain. Man Ther. 2011;16(6):578-84.

24. Bressel E, Dolny DG, Vandenberg C, Cronin JB. Trunk muscle activity during spine stabilization exercises performed in a pool. Phys Ther Sport. 2012;13(2):67-72.

25. Lehman GJ, Gordon T, Langley J, Pemrose P, Tregaskis S. Replacing a Swiss ball for an exercise bench causes variable changes in trunk muscle activity during upper limb strength exercises. Dyn Med. 2005;4:6

26. Vezina MJ, Hubley-Kozey CL. Muscle activation in therapeutic exercises to improve trunk stability. Arch Phys Med Rehabil. 2000;81(10):1370-9.

27. Mayer JM, Graves JE, Clark BC, Formikell M, Ploutz-Snyder LL. The use of magnetic resonance imaging to evaluate lumbar muscle activity during trunk extension exercise at varying intensities. Spine (Phila Pa 1976). 2005;30(22):2556-63.

28. Vera-Garcia FJ, Moreside JM, McGill SM. Abdominal muscle activation changes if the purpose is to control pelvis motion or thorax motion. J Electromyogr Kinesiol. 2011;21(6):893-903.

29. Mew R. Comparison of changes in abdominal muscle thickness between standing and crook lying during active abdominal hollowing using ultrasound imaging. Man Ther. 2009;14(6):690-5.

30. Rasouli O, Arab AM, Amiri M, Jaberzadeh S. Ultrasound measurement of deep abdominal muscle activity in sitting positions with different stability levels in subjects with and without chronic low back pain. Man Ther. 2011;16(4):388-93.

31. Queiroz BC, Cagliari MF, Amorim CF, Sacco IC. Muscle activation during four Pilates core stability exercises in quadruped position. Arch Phys Med Rehabil. 2010;91(1):86-92.

32. Okubo Y, Kaneoka K, Imai A, Shiina I, Tatsumura M, Izumi S, et al. Electromyographic analysis of transversus abdominis and lum bar multifidus using wire electrodes during lumbar stabilization exercises. J Orthop Sports Phys Ther. 2010;40(11):743-50.

33. Morris SL, Lay B, Allison GT. Corset hypothesis rebutted--transversus abdominis does not co-contract in unison prior to rapid arm movements. Clin Biomech (Bristol, Avon). 2012;27(3):249-54.

34. Arab AM, Chehrehrazi M. Ultrasound measurement of abdominal muscles activity during abdominal hollowing and bracing in women with and without stress urinary incontinence. Man Ther 2011;16(6):596-601.

35. Ainscough-Potts AM, Morrissey MC, Critchley D. The response of the transverse abdominis and internal oblique muscles to different postures. Man Ther. 2006;11(1):54-60.

36. Kim Y, Shim JK, Son J, Pyeon HY, Yoon B. A neuromuscular strategy to prevent spinal torsion: backward perturbation alters asymmetry of transversus abdominis muscle thickness into symmetry. Gait Posture. 2013;38(2):231-5.

37. Watanabe $\mathrm{H}$, Abe H, Tojima M, Yoshimoto M, Takahira N, Torii S. Ultrasonography of the deep abdominal muscles at rest and during the drawing-in maneuver: a comparative study of Japanese adolescent vs. adult soccer players. Isokinet Exerc Sci. 2013;21(3):187-93.

38. Allison GT, Morris SL, Lay B. Feedforward responses of transver- 


\section{Emami F et al.}

sus abdominis are directionally specific and act asymmetrically: implications for core stability theories. J Orthop Sports Phys Ther. 2008;38(5):228-37.

39. Yilmaz F, Yilmaz A, Merdol F, Parlar D, Sahin F, Kuran B. Efficacy of dynamic lumbar stabilization exercise in lumbar microdiscectomy.J Rehabil Med. 2003;35(4):163-7.

40. Hides J, Stanton W, Mendis MD, Sexton M. The relationship of transversus abdominis and lumbar multifidus clinical muscle tests in patients with chronic low back pain. Man Ther. 2011;16(6):573-7.

41. Levangie PK, Santasier AM, Stout NL, Pfalzer L. A qualitative assessment of upper quarter dysfunction reported by physical therapists treated for breast cancer or treating breast cancer sequelae. Support Care Cancer. 2011;19(9):1367-78. 